\title{
High proton conduction in grain-boundary-free yttrium-doped barium zirconate films grown by pulsed laser deposition
}

\author{
Daniele Pergolesi ${ }^{1,2 \dagger}$, Emiliana Fabbri ${ }^{1,2 \dagger}$, Alessandra D'Epifanio ${ }^{1}$, Elisabetta Di Bartolomeo', \\ Antonello Tebano ${ }^{3}$, Simone Sanna ${ }^{1}$, Silvia Licoccia ${ }^{1}$, Giuseppe Balestrino ${ }^{3}$ and Enrico Traversa ${ }^{1,2 \star}$
}

\begin{abstract}
Reducing the operating temperature in the $500-750^{\circ} \mathrm{C}$ range is needed for widespread use of solid oxide fuel cells (SOFCs). Proton-conducting oxides are gaining wide interest as electrolyte materials for this aim. We report the fabrication of $\mathrm{BaZr}_{0.8} \mathrm{Y}_{0.2} \mathrm{O}_{3-\delta}$ (BZY) proton-conducting electrolyte thin films by pulsed laser deposition on different single-crystalline substrates. Highly textured, epitaxially oriented BZY films were obtained on (100)-oriented MgO substrates, showing the largest proton conductivity ever reported for BZY samples, being $0.11 \mathrm{~S} \mathrm{~cm}^{-1}$ at $500^{\circ} \mathrm{C}$. The excellent crystalline quality of BZY films allowed for the first time the experimental measurement of the large BZY bulk conductivity above $300{ }^{\circ} \mathrm{C}$, expected in the absence of blocking grain boundaries. The measured proton conductivity is also significantly larger than the conductivity values of oxygen-ion conductors in the same temperature range, opening new potential for the development of miniaturized SOFCs for portable power supply.
\end{abstract}

$\mathrm{R}$ educing the SOFC operating temperature in the intermediate temperature range, $500-750^{\circ} \mathrm{C}$, is one of the major targets in present SOFC research ${ }^{1,2}$, and it is also a requisite for the development of miniaturized SOFCs for portable power supply ${ }^{3,4}$. Improving the electrolyte performance for intermediate-temperature operation can be achieved by reducing the electrolyte thickness ${ }^{5,6}$, and by using alternative materials to yttria-stabilized zirconia with a larger ionic conductivity in the intermediate temperature range ${ }^{7}$. With respect to the oxygen-ion conductors conventionally used in SOFCs, electrolytes based on high-temperature proton conductors (HTPCs) take advantage of their lower activation energy for charge transport ${ }^{8}$ and of water formation at the cathode side ${ }^{9,10}$, thereby resulting in suitable conductivity in the intermediate temperature range and avoiding fuel dilution with water.

Among HTPCs, Y-doped barium cerate (BCY) electrolytes have shown rather high protonic conductivity $\left(10^{-2} \mathrm{~S} \mathrm{~cm}^{-1}\right.$ at $600{ }^{\circ} \mathrm{C}$; ref. 11), although BCY strongly reacts with $\mathrm{CO}_{2}$ (ref. 12) and water vapour $^{13}$, hindering technological applications. On the other hand, despite a very good chemical stability of Y-doped barium zirconate (BZY) under fuel-cell operating environments, the total proton conductivity of BZY sintered pellets is generally significantly lower (about $10^{-3} \mathrm{~S} \mathrm{~cm}^{-1}$ at $600{ }^{\circ} \mathrm{C}$; refs 14,15 ). This is due to the poor sinterability of BZY (ref. 16), together with the poor conducting properties of BZY grain boundary regions ${ }^{17}$. Scattered conductivity values for BZY samples are reported in the literature, and mostly depend on the processing parameters (see Supplementary Fig. S1).

However, about a decade ago electrochemical impedance spectroscopy (EIS) measurements at temperatures below $200^{\circ} \mathrm{C}$, that is, in the temperature range where impedance spectra allowed separation of the bulk and the grain boundary contribution, showed for the first time larger bulk conductivity for BZY sintered pellets than for BCY (ref. 18). Furthermore, theoretical extrapolations in the intermediate-temperature SOFC (IT-SOFC) operating range have suggested that the BZY bulk conductivity might exceed the conductivity of the best oxygen-ion conductors below $700{ }^{\circ} \mathrm{C}$ (ref. 8).

These findings stimulated in the past decade a growing interest in BZY (ref. 19). Several approaches have been reported to tackle the challenge of processing dense BZY electrolyte membranes: in addition to the preparation of BCY-BZY solid solutions to compromise between the two materials properties ${ }^{14,15,20}$, the synthesis of ultrafine BZY powders allowed reduction of the sintering temperature while keeping the grain size small ${ }^{21-23}$, and the use of extreme sintering temperatures ${ }^{24}$ and of sintering aids ${ }^{25,26}$ led to full densification and grain growth but did not improve BZY conductivity owing to a change in the materials stoichiometry. A significant improvement in conductivity has been reported recently for BZY pellets with a grain size larger than $1 \mu \mathrm{m}$, fabricated according to an improved sintering protocol without using sintering aids ${ }^{27}$.

Recently, with the aim of minimizing the grain boundary contribution to the total conductivity, 60-nm-thick highly textured BZY films have been fabricated ${ }^{28}$. Their large conductivity was very close to the reported BZY bulk conductivity ${ }^{18}$, even though the investigated temperature range extended up to no more than $290^{\circ} \mathrm{C}$.

Here, we report the fabrication by pulsed laser deposition (PLD) of highly textured, epitaxially oriented BZY films with a thickness of $1 \mu \mathrm{m}$. The excellent crystalline quality of these films allowed for the first time, to the best of our knowledge, the experimental measurement of the large BZY bulk conductivity expected in the

\footnotetext{
${ }^{1}$ NAST Center \& Dipartimento di Scienze e Tecnologie Chimiche, University of Roma 'Tor Vergata', 00133 Rome, Italy, ${ }^{2}$ International Research Center for Materials Nanoarchitectonics (MANA), National Institute for Materials Science (NIMS), Tsukuba, Ibaraki 305-0044, Japan, ${ }^{3}$ CNR-SPIN and Dipartimento di Ingegneria Meccanica, University of Roma 'Tor Vergata', 00133 Rome, Italy. 'These authors contributed equally to this work. ^e-mail: traversa.enrico@nims.go.jp.
} 

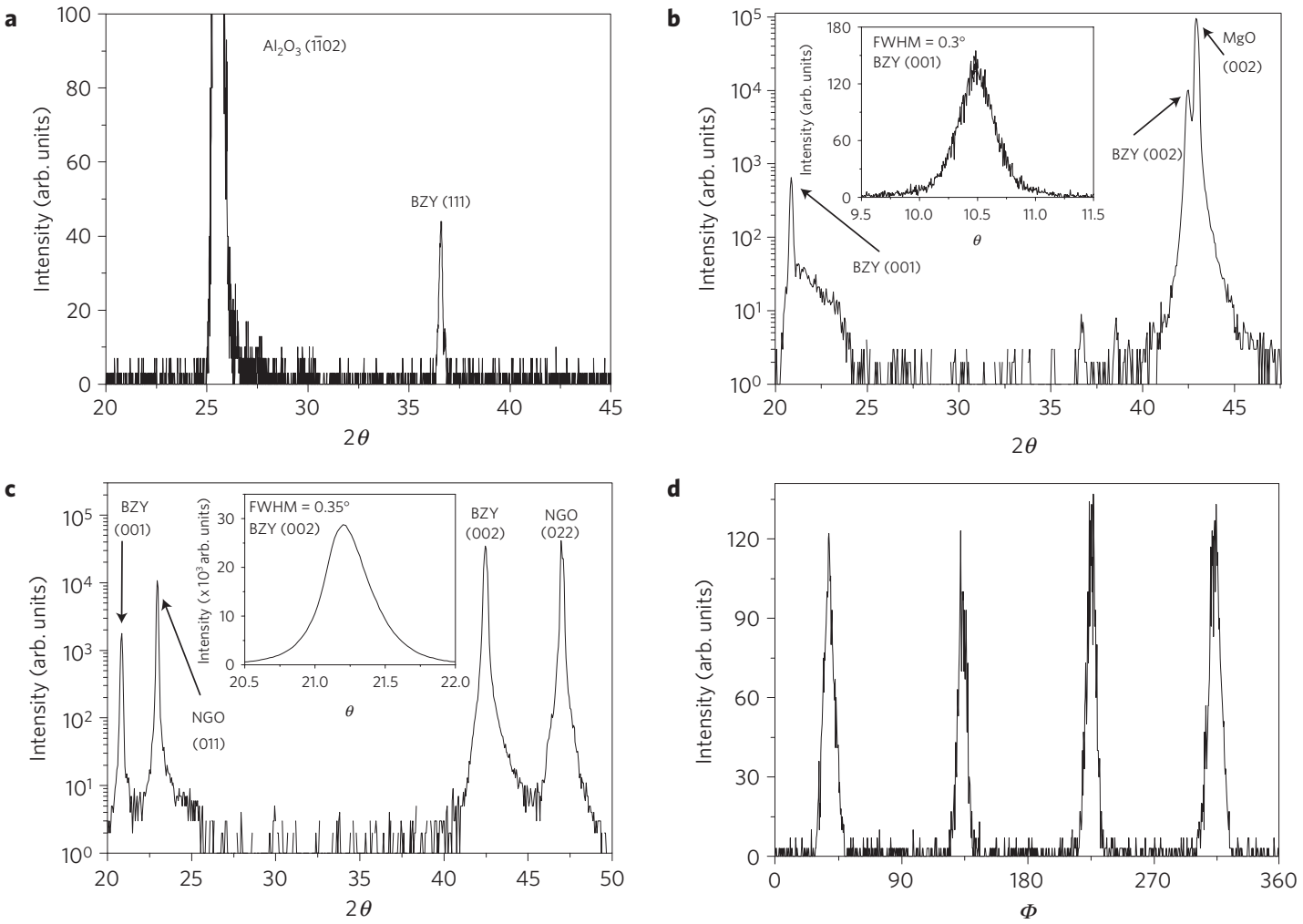

Figure 1 | XRD analysis of BZY films. a-c, XRD pattern of $\mathrm{BZY}$ films grown on R-cut (1102) $\mathrm{Al}_{2} \mathrm{O}_{3}$ (a), (001)-oriented $\mathrm{MgO}$ (b) and (011)-oriented NGO (c). The insets show the rocking curves. BZY grew epitaxially oriented with the deposition substrate in the case of both MgO and NGO, whereas on the hexagonal structure of sapphire it grew in the (111) growth direction. $\mathbf{d}, \phi$-scan of the film grown on NGO, showing a fully relaxed biaxially textured structure.

absence of blocking grain boundaries, but in the temperature range suitable for IT-SOFC operation.

PLD is a flexible technique suitable for the preparation of oxide films. It is gaining increasing attention for the fabrication of SOFC materials $s^{6,28,29}$, because it allows careful control of the crystalline structure $^{30}$ and morphology ${ }^{31}$ of the deposited materials, usually preserving their required stoichiometry.

Several $\mathrm{BaZr}_{0.8} \mathrm{Y}_{0.2} \mathrm{O}_{3-\delta}$ films, approximately $1 \mu \mathrm{m}$ thick, were fabricated by PLD on different single-crystalline substrates. Barium zirconate, $\mathrm{BaZrO}_{3}$, crystallizes in a cubic perovskite structure with a lattice parameter of $4.1973 \AA$, as reported in the ICSD database, no. 90049. The partial substitution of $\mathrm{Y}$ into the $\mathrm{Zr}$ site increases the unit-cell volume, causing local distortions of the lattice ${ }^{32-34}$.

Good crystallographic matching between the film and substrate materials is required for growing highly crystalline films. Different substrates were selected to prepare epitaxial BZY films; (100)oriented $\mathrm{SrTiO}_{3}$ and $\mathrm{LaAlO}_{3}$ cubic perovskite structures $(a=3.90$ and $3.79 \AA$, respectively) and a (110)-oriented $\mathrm{NdGaO}_{3}$ (NGO) orthorhombic perovskite structure $(a=b=3.863 \AA, c=3.854 \AA$ in the pseudocubic cell) were used. These substrates allowed investigation of the BZY film growth mechanisms, but their residual electronic conductivity at high temperatures precludes measuring reliable conductivity values ${ }^{30}$. Two insulating substrates were selected for electrical measurements, single-crystal (100)-oriented $\mathrm{MgO}$ substrates with cubic structure $(a=4.21 \AA)$, having a very good lattice match with BZY, and R-cut (1102)-oriented sapphire substrates with hexagonal structure $(a=b=4.76 \AA, c=12.99 \AA)$, having a larger mismatch with the BZY lattice.

The crystalline structure of the prepared BZY films was investigated by X-ray diffraction (XRD) analysis. Figure 1 shows the XRD plots of $1-\mu \mathrm{m}$-thick BZY films, deposited on sapphire (Fig. 1a), MgO (Fig. 1b) and NGO (Fig. 1c). The XRD plots of the films grown on $\mathrm{SrTiO}_{3}$ and $\mathrm{LaAlO}_{3}$ are not reported because they were very similar to the XRD plots of the BZY films deposited on NGO.

Among the selected substrates, sapphire provided the largest crystallographic mismatch with the BZY cubic perovskite structure (Fig. 1a). Nevertheless, the XRD plot showed that the sapphire substrate drove the BZY film growth towards the (111) direction. The relatively weak intensity of the (111) peak can be attributed to the intrinsically small relative intensity of this reflection ${ }^{14,16,21,24-26}$. The angular position of the BZY (111) reflection line was consistent with an out-of-plane lattice constant of about $4.25 \AA$. The weak intensity did not allow measurement of the rocking curve in a reliable way.

The BZY films grown on both $\mathrm{MgO}$ (Fig. 1b) and cubic perovskite crystals (Fig. 1c) were (100)-epitaxially oriented. Despite the strong overlap of BZY and $\mathrm{MgO}$ (200) reflection lines (Fig. 1b), owing to the very close lattice parameters, the reflection peaks of the BZY film are distinct from the substrate peaks and their identification is unambiguous. However, their intensity ratio and the narrow spread in their angular positions preclude an accurate measurement of the rocking curves of the BZY peak on $\mathrm{MgO}$. Thus, although affected by larger errors, the rocking curve of the (100) reflection was measured, showing a full-width at half-maximum (FWHM) of $0.3^{\circ}$ (inset in Fig. 1b). For the films deposited on NGO, the rocking curve of the BZY (200) reflection (inset in Fig. 1c) showed a FWHM of $0.35^{\circ}$. Intrinsic restrictions to the crystalline quality of the films can be expected owing to the relatively low deposition temperature $\left(600^{\circ} \mathrm{C}\right)$, the large dopant content $(20 \%)$ and the large thickness (about $1 \mu \mathrm{m}$ ).

The measured out-of-plane lattice parameter was about 4.22-4.23 $\AA$ for BZY films grown either on $\mathrm{MgO}$ or cubic perovskite substrates. Despite the relatively large crystallographic 
$\mathbf{a}$
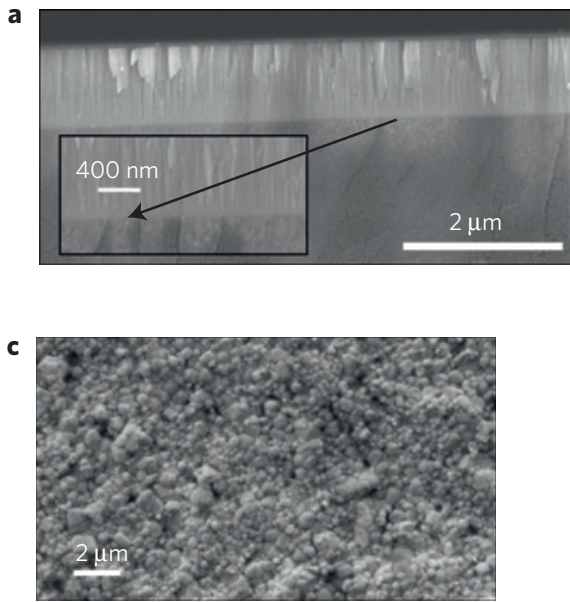

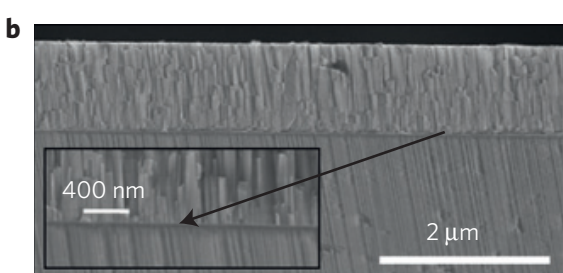

d

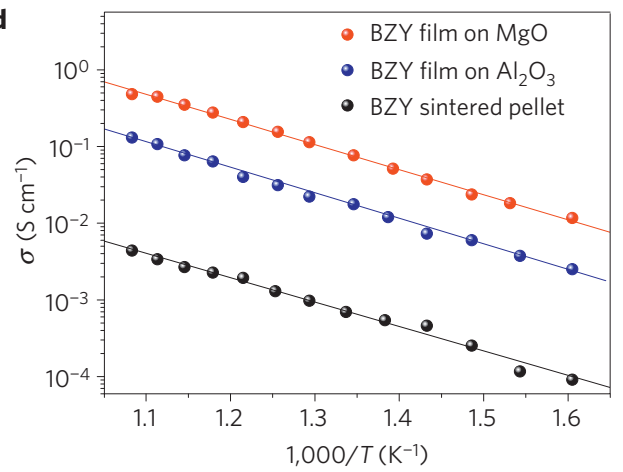

Figure 2 | Morphology influence on conductivity. a-c, Cross-section FE-SEM micrograph of a BZY film on MgO (a), a BZY film on sapphire (b) and a BZY sintered pellet (c). The cross-section samples were obtained after fracturing; thus, the features shown in $\mathbf{a}$ and $\mathbf{b}$ can be ascribed to cleavage planes. $\mathbf{d}$, The Arrhenius plots of the conductivity measured in a wet $5 \% \mathrm{H}_{2}$ in Ar atmosphere for the three samples. For the films, two parallel strip-shaped Pt electrodes, a few tens of nanometres thick, were deposited by PLD on the film surface at a distance of $1 \mathrm{~mm}$, and wired to the read-out electronics using Pt paste and wires. A large impact of the BZY morphology on its electrical properties is observed. The highly textured BZY film grown on the MgO substrate presents conductivity values about two orders of magnitude larger than the total conductivity of sintered pellets of the same material.

mismatch of the perovskite substrates, BZY films are thick enough to allow relaxation of the crystalline structure above the substrate-to-film interface.

The 'in-plane' crystalline structure of the films grown on perovskite substrates was investigated by XRD analysis. Figure 1d shows the $\phi$-scan measurements carried out towards the (103) asymmetric reflection of the BZY films grown on NGO, revealing the biaxially textured structure.

The BZY films grown on $\mathrm{MgO}$ were also analysed by in situ reflection high energy electron diffraction (RHEED). The resulting pattern of the film, measured at the end of the deposition, showed the typical features of the BZY perovskite structure, demonstrating the quasi-ideal two-dimensional film growth with cube-on-cube orientation. Furthermore, the film in-plane lattice parameters were the same as for the substrate (see Supplementary SI, Fig. S2).

To further investigate the epitaxial strain of the BZY films grown on $\mathrm{MgO}$, reciprocal space maps were recorded around the (204) diffraction peak of the $\mathrm{MgO}$ substrate. From the map, a value of about $4.21 \AA$ can be calculated for the in-plane film lattice parameter. On the other hand, the out-of-plane lattice parameter was about $4.23 \AA$, in agreement with the standard $\theta-2 \theta$ symmetric scan measurements. Therefore, the in-plane lattice parameter matched well the $\mathrm{MgO}$ lattice parameter, whereas the slightly larger out-of-plane lattice parameter may be due to a small epitaxial strain in the film crystallographic structure (see Supplementary SI, Fig. S3).

X-ray photoelectron spectroscopy (XPS) measurements did not reveal any sign of chemical contamination and showed a substantial stoichiometric equivalence between the chemical compositions of the films and the ablation target. Moreover, confirming the XRD results, the analysis of the binding-energy chemical shift of the different elements did not reveal the presence of other compounds except for BZY (see Supplementary SII).

Field-emission scanning electron microscopy (FE-SEM) observations of the surface of $\mathrm{BZY}$ films grown on $\mathrm{MgO}$ and sapphire did not show the presence of any features. Figure 2 shows the typical FE-SEM micrographs of the cross-sections of the BZY films grown on $\mathrm{MgO}$ (Fig. 2a) and sapphire (Fig. 2b). A fully dense microstructure without grains was observed for the films grown on both substrates. Both films showed a good adhesion to the substrates and the interfaces are sharp and clean. It is worth noting that as-deposited dense films are grown by PLD already at $600^{\circ} \mathrm{C}$, a temperature that is much lower than the conventional BZY sintering temperature, without the need of post-annealing treatments.

For comparison, Fig. $2 c$ shows the morphology of the fracture surface of a BZY pellet sintered at $1,600^{\circ} \mathrm{C}$ for $8 \mathrm{~h}$, as described elsewhere $^{21}$. In agreement with several literature reports, the pellet showed an average grain size smaller than $1 \mu \mathrm{m}$ and a relative density of about $90 \%$ of the theoretical density.

EIS measurements were used to evaluate the conducting properties of the BZY samples as a function of temperature. For the films, two parallel strip-shaped Pt electrodes were deposited by PLD on the film surface at a distance of $1 \mathrm{~mm}$. The conductivity was measured in the $350-650{ }^{\circ} \mathrm{C}$ temperature range, in a humid atmosphere (about 0.03 atm $p_{\mathrm{H}_{2} \mathrm{O}}$ ) of $5 \% \mathrm{H}_{2}$ in Ar. Figure $2 \mathrm{~d}$ shows the Arrhenius plots of the measured conductivity of two samples grown on $\mathrm{MgO}$ and sapphire. For comparison, Fig. $2 \mathrm{~d}$ also reports the electrical conductivity of the BZY sintered pellet shown in Fig. 2c, measured in the same experimental conditions.

The proton conductivity of the $\mathrm{BZY}$ films grown on $\mathrm{MgO}$ swept between $1.2 \times 10^{-2}$ and $5 \times 10^{-1} \mathrm{~S} \mathrm{~cm}^{-1}$ in the temperature range tested. These conductivity values are about two orders of magnitude larger than the BZY sintered pellet conductivity. The proton conductivity of the BZY films grown on sapphire turned out to be approximately fourfold smaller than the conductivity of the films grown on $\mathrm{MgO}$, but 20 times larger than the conductivity of the sintered pellets. The measured activation energy for proton conduction was about $0.63 \mathrm{eV}$ for films grown on both $\mathrm{MgO}$ and sapphire, larger than the $0.48 \mathrm{eV}$ reported in the literature for bulk conductivity of a BZY pellet ${ }^{32}$, measured though below $150{ }^{\circ} \mathrm{C}$. These findings, together with the larger pre-exponential factor of the conductivity and the absence of dehydration up to $600^{\circ} \mathrm{C}$, 
a

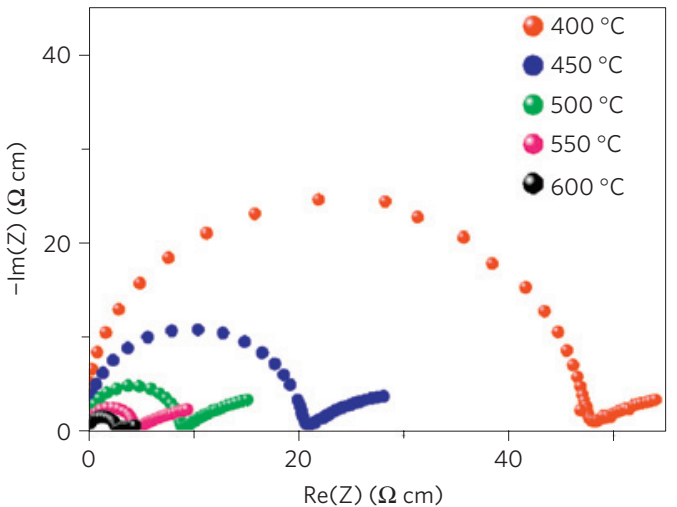

c

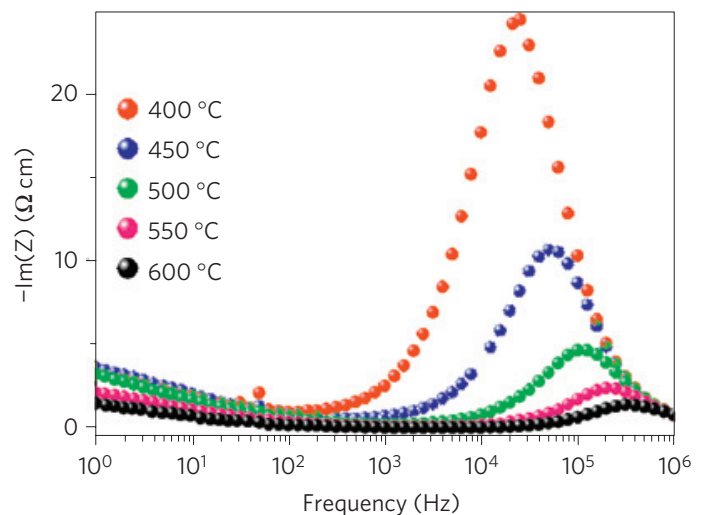

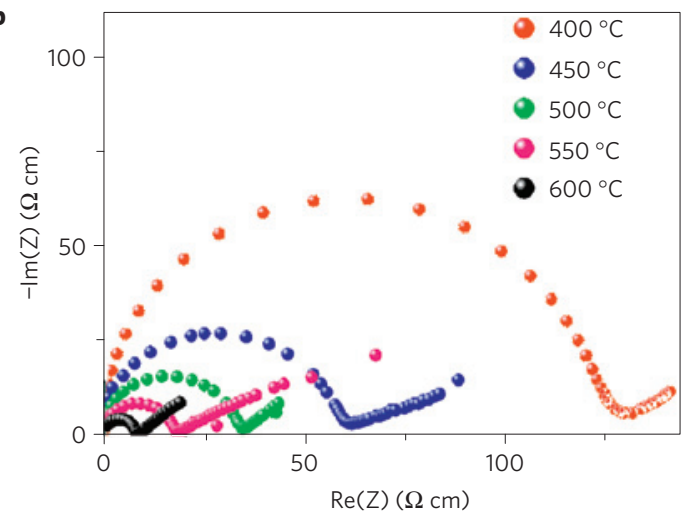

d

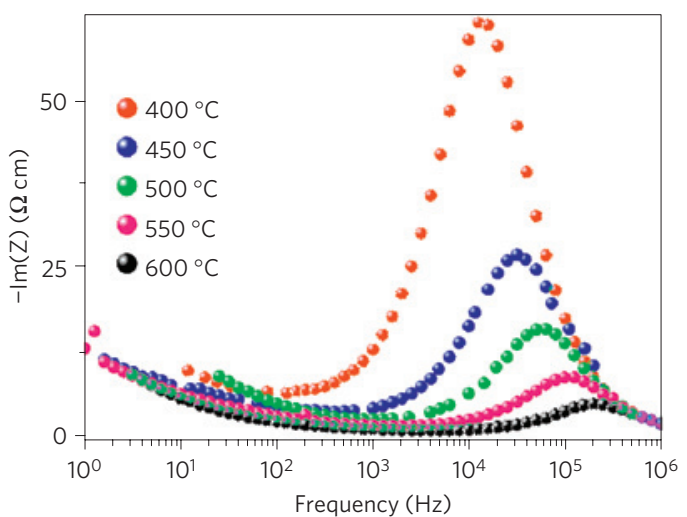

Figure 3 | EIS analysis of BZY films. a,b, Nyquist plots measured between 400 and $600{ }^{\circ} \mathrm{C}$ in a humidified $5 \%$ H in Ar atmosphere for $1-\mu m$-thick $\mathrm{BZY}$ films deposited on $\mathrm{MgO}(\mathbf{a})$ and $\mathrm{Al}_{2} \mathrm{O}_{3}$ (b). Single semiarcs at high frequencies, together with electrode polarization at low frequencies, appeared in the complex impedance-plane plots of both BZY films grown on MgO and sapphire substrates. c,d, The spectroscopic plots of the impedance imaginary part for the BZY films grown on $\mathrm{MgO}$ (c) and sapphire (d), confirming the presence of a single peak at high frequencies.

show that structural and/or chemical differences exist between the samples prepared using PLD and ceramic processing, even on the bulk level. For the latter, dehydration was observed to start at about $400^{\circ} \mathrm{C}$ and a water partial pressure of $2 \mathrm{kPa}$ (ref. 32).

The only differences between the two BZY films are their crystalline orientation, as revealed by XRD analysis, and the crystalline mismatch with the deposition substrate, which was larger for the BZY films deposited on sapphire, always considering the excellent crystalline quality of both films. Therefore, these are the factors that can explain the difference in proton conductivity between the films. Given that the activation energy was the same, crystalline orientation and mismatch degree seem to affect only the pre-exponential factor.

The values reported in the Arrhenius plots were derived from the intercepts of the semicircle in the complex impedance-plane plots with the real axis, owing to the much larger conductivity of the films with respect to the conductivity of the insulating substrates. Differently, the measured capacitance cannot be attributed to the film owing to the interfering substrate geometric capacitance and measuring set-up stray capacitance. Figure 3 shows the Nyquist plots of the BZY films; at high frequencies a single semicircle, with a negligible depression, could be observed up to $650^{\circ} \mathrm{C}$ for both the BZY films grown on $\mathrm{MgO}$ (Fig. 3a) and sapphire (Fig. 3b). In contrast, the reported high-frequency region of Nyquist plots for BZY pellets usually shows a depressed semicircle owing to the overlapping of several $\operatorname{arcs}^{25,35}$, which were disentangled only below $300^{\circ} \mathrm{C}$ (refs $18,27,36,37$ ).

At low frequencies, a linear spike was observed above $400{ }^{\circ} \mathrm{C}$, attributable to electrode polarization. The blocking effect of the $\mathrm{Pt}$ electrodes suggested the absence of electron conductivity. Further confirmation that the conductivity was mostly due to proton charge carriers was obtained from EIS measurements carried out in a $\mathrm{D}_{2} \mathrm{O}$ containing Ar atmosphere (see Supplementary SIII). The possibility of a parallel surface conduction path was ruled out by measuring films with different thicknesses (see Supplementary SIV).

Figure 3 shows also the spectroscopic plots of the impedance imaginary part for the BZY films grown on $\mathrm{MgO}$ (Fig. 3c) and sapphire (Fig. 3d). These plots confirmed the presence of a single peak at high frequencies, clearly indicating the presence of a single conduction mechanism. Furthermore, the peaks were symmetrical, demonstrating the absence of distortion resulting from inductive effects.

The charge carriers in a HTPC depend on the temperature, atmosphere, oxygen partial pressure and water partial pressure ${ }^{38,39}$. Therefore, EIS measurements were also carried out in a dry Ar atmosphere, where oxygen-ion conduction should take place, and in a dry $\mathrm{O}_{2}$ atmosphere, where mixed ionic-electronic conduction should prevail. Figure $4 \mathrm{a}$ shows the Arrhenius plots measured in wet $5 \% \mathrm{H}_{2}$ in $\mathrm{Ar}$, dry $\mathrm{O}_{2}$ and dry $\mathrm{Ar}$ atmospheres for the BZY film deposited on $\mathrm{MgO}$. Below $600^{\circ} \mathrm{C}$, the conductivity in the humidified atmosphere was larger than that in both dry atmospheres, in agreement with other studies on protonconducting crystals ${ }^{40,41}$. The significant increase in the activation energy under dry conditions, compared with wet conditions, clearly indicated that the nature of the charge carriers changed ${ }^{42}$. As observed for the measurements in the wet environment (Fig. 2d), the conductivity values measured in dry Ar and dry $\mathrm{O}_{2}$ are also about two orders of magnitude larger than the conductivity values measured in the same environments for BZY sintered pellets ${ }^{43}$.

Figure $4 \mathrm{~b}$ and $\mathrm{c}$ show the complex impedance-plane plots for the $\mathrm{BZY}$ film deposited on $\mathrm{MgO}$ measured at $500^{\circ} \mathrm{C}$ in dry $\mathrm{O}_{2}$ and dry 

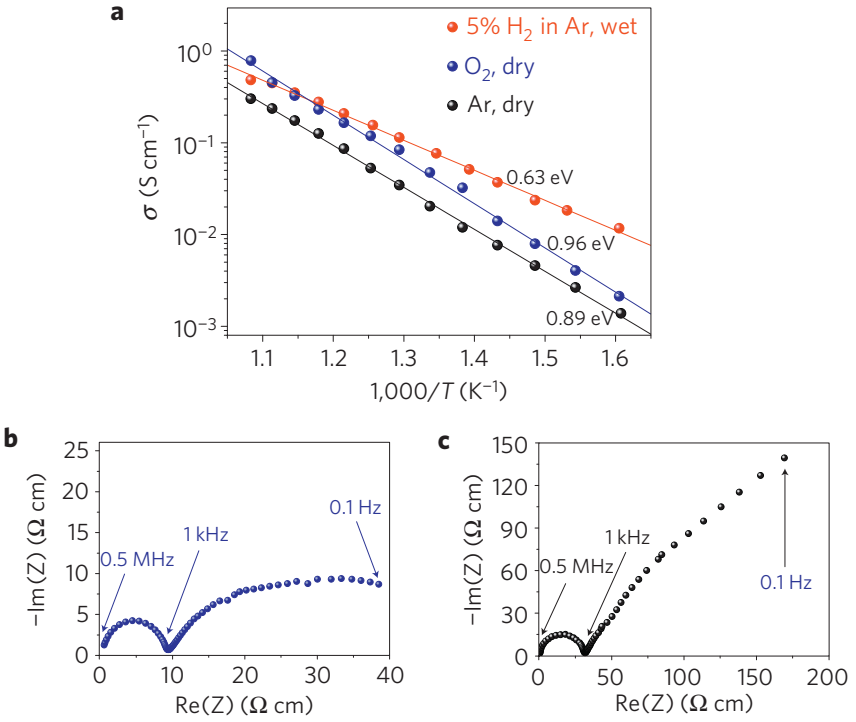

Figure 4 | Arrhenius plots of BZY film conductivity in different atmospheres. a, Arrhenius plots of the conductivity of BZY films grown on $\mathrm{MgO}$ measured in wet $5 \% \mathrm{H}_{2}$ in $\mathrm{Ar}$, dry $\mathrm{O}_{2}$ and dry $\mathrm{Ar}$ atmospheres. Different conduction mechanisms take place in these environments. Protons, electron-holes and oxygen ions are the main charge carriers in wet $5 \% \mathrm{H}_{2}$ in $\mathrm{Ar}$, dry $\mathrm{O}_{2}$ and dry Ar atmospheres, respectively. As further evidence of the different conduction mechanisms, the complex impedance-plane plots for the BZY film deposited on $\mathrm{MgO}$ measured at $500^{\circ} \mathrm{C} \mathrm{(b)} \mathrm{in} \mathrm{dry} \mathrm{O}_{2}$ and (c) dry Ar atmospheres are reported. The low-frequency part, characteristic of the Pt/BZY interface, showed significant differences. The plot of the film measured in dry $\mathrm{O}_{2}(\mathbf{b})$ showed a semiarc that tends to close on the real axis, showing the presence of a more important electronic contribution. On the other hand, the plot of the samples measured in $\operatorname{dry} \operatorname{Ar}(\mathbf{c})$ showed a spike almost at $45^{\circ}$, showing a larger polarization effect than for the case of the measurements in a humid environment (Fig. 3a). Therefore, the polarization effects decrease in the order: dry $\mathrm{Ar}>$ wet $5 \% \mathrm{H}_{2}$ in $\mathrm{Ar}>$ dry $\mathrm{O}_{2}$.

Ar atmospheres, respectively. Also, in both dry environments the high-frequency region showed the presence of a single semicircle. Instead, the low-frequency part, characteristic of the Pt/BZY interface, showed significant differences in the polarization effects, which decrease in the order: dry $\mathrm{Ar}>$ wet $5 \% \mathrm{H}_{2}-\mathrm{Ar}>\operatorname{dry} \mathrm{O}_{2}$. This is consistent with the charge transfer reaction of oxygen ions, the major charge carriers in dry Ar, which is more difficult than for protons, the major charge carriers in wet environments, and a larger presence of electronic conduction accounts for the reduced polarization in dry $\mathrm{O}_{2}$.

The very large proton conductivity measured for the BZY films deposited on $\mathrm{MgO}$ substrates is compatible with the lowtemperature measurements reported in refs 8,32, as shown in Fig. 5a. Therefore, the marked increase in conductivity for the films with respect to BZY sintered pellets can be ascribed to the high crystalline quality of the epitaxial films that minimizes non-conductive grain boundary regions, which instead dominate in sintered pellets. These results indeed fulfilled the suggestion to study $\mathrm{BZY}$ in the form of epitaxial films to fully exploit its potential ${ }^{17}$. Our measurements did not confirm the recent hypothesis that an increase in BZY grain size above $1 \mu \mathrm{m}$ would provide only a marginal increase in total conductivity ${ }^{27}$. To the best of our knowledge, the reported conductivity values can be considered as the best approximation to the direct measurement of the BZY bulk conductivity at high temperatures.

The observed boost in proton conductivity for the BZY films highlights the crucial relevance of careful control of the electrolyte

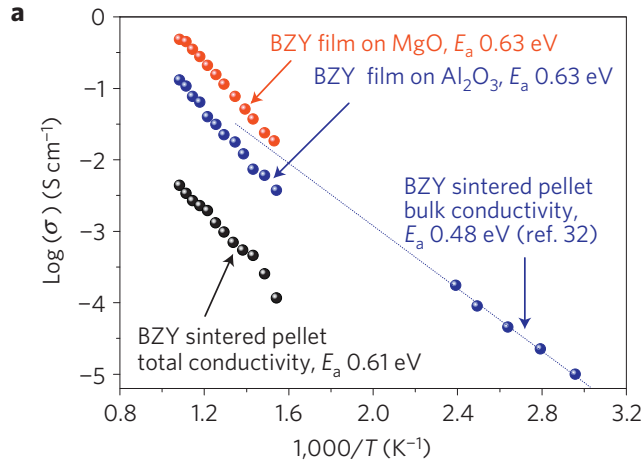

b

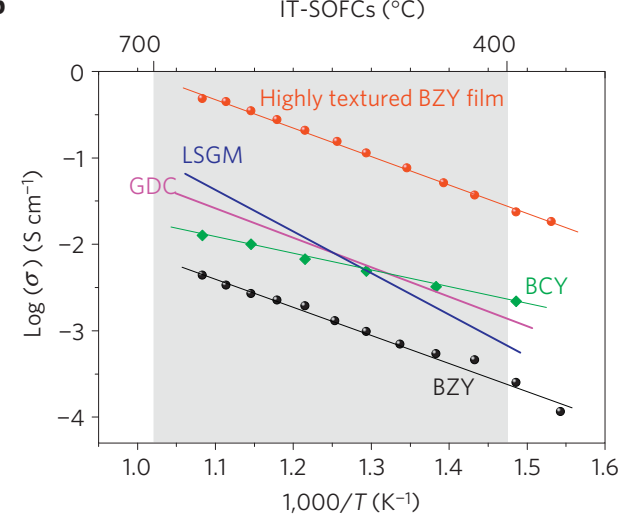

Figure 5 | Electrical conductivity comparisons. a, Comparison between the electrical conductivity and activation energy values of the BZY films grown on $\mathrm{MgO}$ and sapphire, and of BZY sintered pellets, measured in the intermediate temperature range, with the bulk conductivity values of BZY pellets measured at low temperature ${ }^{32}$. The differences in the measured activation energy values from the literature data indicate some structural and/or chemical differences between the bulk of the samples prepared using PLD and ceramic processing. $\mathbf{b}$, Comparison between the electrical conductivity values of the $\mathrm{BZY}$ film grown on $\mathrm{MgO}$, and of $\mathrm{BZY}$ and $\mathrm{BCY}$ sintered pellets, measured in the intermediate temperature range ${ }^{14}$ Conductivity values of the best-performing oxygen-ion-conducting materials, $\mathrm{La}_{0.8} \mathrm{Sr}_{0.2} \mathrm{Ga}_{0.8} \mathrm{Mg}_{0.2} \mathrm{O}_{3}$ (LSGM; ref. 46) and $\mathrm{Ce}_{0.8} \mathrm{Gd}_{0.2} \mathrm{O}_{1.9-\delta}$ (GDC; ref. 47), are also reported.

crystalline structure, which can be achieved by PLD processing. Indeed, the epitaxial films are a powerful tool for studying the BZY grain bulk conduction, but the potential interest for practical application in miniaturized SOFCs (ref. 44) should not be neglected. Preliminary tests were carried out using BZY films grown on $\mathrm{MgO}$ as fuel-cell electrolytes. Owing to the presence of the $\mathrm{MgO}$ substrate, a single-chamber configuration was mandatory to carry out these preliminary tests. Despite the very low open-circuit voltage resulting from the severely unfavourable electrochemical design ${ }^{45}$, the single-chamber cell was able to supply a shortcircuit current density one order of magnitude larger than the value measured for the sintered BZY pellet tested in a doublechamber configuration $^{21}$ (see Supplementary SV). This is strong evidence of the excellent performance of the BZY film electrolyte, notwithstanding the far-from-good electrode performance.

The comparison with literature data showed that not only the measured conductivity of the highly textured BZY films grown on $\mathrm{MgO}$ substrates was the largest ever reported for BZY samples (see Supplementary Fig. S9), but in the intermediate operating temperature range for SOFCs their conductivity is significantly larger than the reported values for the best-performing, stable oxygen-ion conductors, as shown in Fig. 5b. The chemical stability of BZY, together with the very large proton conductivity verified in 
this work, certainly demonstrates that BZY films without blocking grain boundaries are amongst the best-performing electrolytes ever developed for possible fuel-cell applications.

\section{Methods}

The PLD (equipment assembled in the laboratory) system consisted of a vacuum chamber equipped with a turbo molecular pump, with a base pressure of about $10^{-4} \mathrm{~Pa}$. A gas inlet line allowed setting of the required oxygen partial pressure during the deposition. A KrF excimer laser (Coherent Lambda Physik GmbH) with a wavelength of $248 \mathrm{~nm}$ and a pulse width of $25 \mathrm{~ns}$ was focused on the target material in a spot area of about $5 \mathrm{~mm}^{2}$. The pulsed laser was used to ablate $20 \mathrm{at} \%$ Y-doped $\mathrm{BaZrO}_{3}$ (BZY) pellets, prepared according to the procedure described elsewhere ${ }^{21}$. A radiant heater was used to set the temperature of the substrate holder up to $600^{\circ} \mathrm{C}$ during the deposition. The thermal contact between the sample holder and the deposition substrate was provided by Ag paste. The laser energy density was about $3 \mathrm{~J} \mathrm{~cm}^{-2}$ with a repetition rate of $10 \mathrm{~Hz}$, producing an ablation rate of about $2.7 \AA \mathrm{s}^{-1}$. The target-to-substrate distance was $40 \mathrm{~mm}$, and the oxygen partial pressure was $5 \mathrm{~Pa}$.

XRD (PANalytical X'pert Pro MPD) analysis was used to investigate the crystalline structure of the films. $2 \theta-\omega$-scans, rocking curves, $\phi$-scans and reciprocal space maps were acquired. The 'in-plane' XRD analysis was carried out towards the (103) asymmetric reflection of the substrate (for $\mathrm{NdGaO}_{3}, 2 \theta=78.02^{\circ}$ and $\left.\omega=20.57^{\circ}\right)$. The same asymmetric reflection was used for the BZY films $\left(2 \theta=70.13^{\circ}\right.$ and $\left.\omega=16.65^{\circ}\right)$. Reciprocal space maps were recorded around the (204) diffraction peak of the $\mathrm{MgO}$ substrate.

FE-SEM (Leo Supra 35) observations were carried out for morphological characterization of sample surface and cross-sections.

For the electrical measurements, two parallel strip-shaped Pt electrodes, a few tens of nanometres thick, were deposited in vacuum, at $500^{\circ} \mathrm{C}$, by PLD on the film surface at a distance of $1 \mathrm{~mm}$, and wired to the read-out electronics using $\mathrm{Pt}$ paste and wires. The EIS measurements were carried out using a multichannel potentiostat VMP3 (Bio-Logic), in wet (about 0.03 atm of $p_{\mathrm{H}_{2} \mathrm{O}}$ ) $5 \% \mathrm{H}_{2}$ in $\mathrm{Ar}$, between $1 \mathrm{MHz}$ and $100 \mathrm{mHz}$, varying the temperature between 350 and $650{ }^{\circ} \mathrm{C}$ An excitation bias voltage from $1 \mathrm{~V}$ down to $200 \mathrm{mV}$ was applied, without observing any significant influence in the high-frequency region of the EIS plots. The charge carriers' nature and their relative influence on the charge transport through the BZY films were studied by acquiring several EIS spectra in dry environments $\left(\mathrm{O}_{2}\right.$ and $\left.\mathrm{Ar}\right)$

\section{Received 7 September 2009; accepted 15 July 2010; published online 19 September 2010}

\section{References}

1. Boudghene Stambouli, A. \& Traversa, E. Solid oxide fuel cells (SOFCs): A review of an environmentally clean and efficient source of energy. Renew. Sustain. Energy Rev. 6, 433-455 (2002).

2. Brett, D. J. L., Atkinson, A., Brandon, N. P. \& Skinner, S. J. Intermediate temperature solid oxide fuel cells. Chem. Soc. Rev. 37, 1568-1578 (2008).

3. Kendall, K. \& Palin, M. A small solid oxide fuel cell demonstrator for microelectronic application. J. Power Sources 71, 268-270 (1998).

4. Perednis, D. \& Gauckler, L. J. Solid oxide fuel cells with electrolytes prepared via spray pyrolysis. Solid State Ion. 166, 229-239 (2004).

5. De Souza, S., Visco, S. J. \& De Jonghe, L. C. Thin-film solid oxide fuel cell with high performance at low temperature. Solid State Ion. 98, 57-61 (1997).

6. Beckel, D. J. et al. Thin films for micro solid oxide fuel cells. J. Power Sources 173, 325-345 (2007).

7. Steele, B. C. H. \& Heinzel, A. Materials for fuel-cell technologies. Nature 414, 345-352 (2001).

8. Kreuer, K. D. Proton-conducting oxides. Annu. Rev. Mater. Res. 33, 333-359 (2003).

9. Bonanos, N. Transport properties and conduction mechanism in high-temperature protonic conductors. Solid State Ion. 53-56, 967-974 (1992).

10. Iwahara, H., Asakura, Y., Katahira, K. \& Tanaka, M. Prospect of hydrogen technology using proton-conducting ceramics. Solid State Ion. 168, 299-310 (2004).

11. Ma, G., Shimura, T. \& Iwahara, H. Ionic conduction and nonstoichiometry in $\mathrm{Ba}_{x} \mathrm{Ce}_{0.9} \mathrm{Y}_{0.1} \mathrm{O}_{3-\alpha}$. Solid State Ion. 110, 103-110 (1998).

12. Zakowsky, N., Williamson, S. \& Irvine, J. T. S. Elaboration of $\mathrm{CO}_{2}$ tolerance limits of $\mathrm{BaCe}_{0.9} \mathrm{Y}_{0.1} \mathrm{O}_{3-\delta}$ electrolytes for fuel cells and other applications. Solid State Ion. 176, 3019-3026 (2005).

13. Bhide, S. V. \& Virkar, A. V. J. Stability of $\mathrm{BaCeO}_{3}$-based proton conductors in water-containing atmospheres. J. Electrochem. Soc. 146, 2038-2044 (1999).

14. Fabbri, E., D’Epifanio, A., Di Bartolomeo, E., Licoccia, S. \& Traversa, E. Tailoring the chemical stability of $\mathrm{Ba}\left(\mathrm{Ce}_{0.8-x} \mathrm{Zr}_{x}\right) \mathrm{Y}_{0.2} \mathrm{O}_{3-\delta}$ protonic conductors for intermediate temperature solid oxide fuel cells (IT-SOFCs). Solid State Ion 179, 558-564 (2008)

15. Katahira, K., Kohchi, Y., Shimura, T. \& Iwahara, H. Protonic conduction in Zr-substituted $\mathrm{BaCeO}_{3}$. Solid State Ion. 138, 91-98 (2000).
16. Babilo, P. \& Haile, S. M. Enhanced sintering of yttrium-doped barium zirconate by addition of ZnO. J. Am. Ceram. Soc. 88, 2362-2368 (2005).

17. Bohn, H. G. \& Schober, T. J. Electrical conductivity of the high-temperature proton conductor $\mathrm{BaZr}_{09} \mathrm{Y}_{011} \mathrm{O}_{295}$. J. Am. Ceram. Soc. 83, 768-772 (2000).

18. Kreuer, K. D. Aspects of the formation and mobility of protonic charge carriers and the stability of perovskite-type oxides. Solid State Ion. 125, 285-302 (1999).

19. Fabbri, E., Pergolesi, D. \& Traversa, E. Materials challenges toward proton conducting oxide fuel cells: A critical review. Chem. Soc. Rev. doi:10.1039/b902343g (2010)

20. Zuo, C., Zha, S., Liu, M., Hatano, M. \& Uchiyama, M. Ba( $\left.\mathrm{Zr}_{0.1} \mathrm{Ce}_{0.7} \mathrm{Y}_{0.2}\right) \mathrm{O}_{3-\delta}$ as an electrolyte for low-temperature solid-oxide fuel cells. Adv. Mater. 18, 3318-3320 (2006).

21. D’Epifanio, A., Fabbri, E., Di Bartolomeo, E., Licoccia, S. \& Traversa, E. Design of $\mathrm{BaZr}_{0.8} \mathrm{Y}_{0.2} \mathrm{O}_{3-\delta}$ protonic conductor to improve the electrochemical performance in intermediate temperature solid oxide fuel cells (IT-SOFCs). Fuel Cells 8, 69-76 (2008).

22. Haile, S. M., Staneff, G. \& Ryu, K. H. Non-stoichiometry, grain boundary transport and chemical stability of proton conducting perovskites. J. Mater. Sci. 36, 1149-1160 (2001)

23. Cervera, R. B., Oyama, Y. \& Yamaguchi, S. Low temperature synthesis of nanocrystalline proton conducting $\mathrm{BaZr}_{0.8} \mathrm{Y}_{0.2} \mathrm{O}_{3-\delta}$ by sol-gel method. Solid State Ion. 178, 569-574 (2007).

24. Duval, S. B. C. et al. Electrical conductivity of the proton conductor $\mathrm{BaZr}_{0.9} \mathrm{Y}_{0.1} \mathrm{O}_{3-\delta}$ obtained by high temperature annealing. Solid State Ion. 178, 1437-1441 (2007)

25. Babilo, P. \& Haile, S. M. Enhanced sintering of yttrium-doped barium zirconate by addition of ZnO. J. Am. Ceram. Soc. 88, 2362-2368 (2005).

26. Duval, S. B. C., Holtappels, P., Stimming, U. \& Graule, T. Effect of minor element addition on the electrical properties of $\mathrm{BaZr}_{0.9} \mathrm{Y}_{0.1} \mathrm{O}_{3-\delta}$. Solid State Ion. 179, 1112-1115 (2008).

27. Yamazaki, Y., Hernandez-Sanchez, R. \& Haile, S. M. High total proton conductivity in large-grained yttrium-doped barium zirconate. Chem. Mater. 21, 2755-2762 (2009)

28. Shim, J. H., Gür, T. M. \& Prinz, F. B. Proton conduction in thin film yttrium-doped barium zirconate. Appl. Phys. Lett. 92, 253115 (2008).

29. Pergolesi, D., Fabbri, E. \& Traversa, E. Chemically stable anode-supported solid oxide fuel cells based on Y-doped barium zirconate thin films having improved performance. Electrochem. Commun. 12, 977-980 (2010).

30. Sanna, S. et al. Fabrication and electrochemical properties of epitaxial samarium-doped ceria films on $\mathrm{SrTiO}_{3}$-buffered $\mathrm{MgO}$ substrates. Adv. Funct. Mater. 19, 1713-1719 (2009).

31. Yoon, J. et al. Nanostructured cathode thin films with vertically-aligned nanopores for thin film SOFC and their characteristics. Appl. Surf. Sci. 254, 266-269 (2007).

32. Kreuer, K. D. et al. Proton conducting alkaline earth zirconates and titanates for high drain electrochemical applications. Solid State Ion. 145, 295-306 (2001).

33. Yamazaki, Y., Babilo, P. \& Haile, S. M. Defect chemistry of yttrium-doped barium zirconate: A thermodynamic analysis of water uptake. Chem. Mater. 20, 6352-26357 (2008)

34. Fabbri, E., Pergolesi, D., Licoccia, S. \& Traversa, E. Does the increase in $\mathrm{Y}$-dopant concentration improve the proton conductivity of $\mathrm{BaZr}_{1-x} \mathrm{Y}_{x} \mathrm{O}_{3-\delta}$ fuel cell electrolytes? Solid State Ion. 181, 1043-1051 (2010).

35. Tao, S. \& Irvine, J. T. S. Conductivity studies of dense yttrium-doped $\mathrm{BaZrO}_{3}$ sintered at $1325^{\circ}$ C. J. Solid State Chem. 180, 3493-3503 (2007).

36. Iguchi, F., Sata, N., Tsurui, T. \& Yugami, H. Microstructures and grain boundary conductivity of $\mathrm{BaZr}_{1-x} \mathrm{Y}_{x} \mathrm{O}_{3}(x=0.05,0.10,0.15)$ ceramics. Solid State Ion. 178, 691-695 (2007).

37. Ahmed, I. et al. Crystal structure and proton conductivity of $\mathrm{BaZr}_{09} \mathrm{Sc}_{0.1} \mathrm{O}_{3-\delta}$. J. Am. Ceram. Soc. 91, 3039-3044 (2008).

38. Bonanos, N., Ellis, B., Knight, K. S. \& Mahmood, M. N. Ionic conductivity of gadolinium-doped barium cerate perovskites. Solid State Ion. 35, 179-188 (1989)

39. Fabbri, E., Oh, T., Licoccia, S., Traversa, E. \& Wachsman, E. D. Mixed protonic/electronic conductor cathodes for intermediate temperature SOFCs based on proton conducting electrolytes. J. Electrochem. Soc. 156, B38-B45 (2009).

40. Muller, J., Kreuer, K. D., Maier, J., Matsuo, S. \& Ishigame, M. A conductivity and thermal gravimetric analysis of a Y-doped $\mathrm{SrZrO}_{3}$ single crystal. Solid State Ion. 97, 421-427 (1997).

41. Higuchi, T. et al. Protonic conduction in the single crystals of $\mathrm{SrZr}_{0.95} \mathrm{M}_{0.05} \mathrm{O}_{3}$ $(\mathrm{M}=\mathrm{Y}, \mathrm{Sc}, \mathrm{Yb}, \mathrm{Er})$. J. Appl. Phys. 40, 4162-4163 (2001).

42. Wang, W. \& Virkar, A. V. Ionic and electron-hole conduction in $\mathrm{BaZr}_{0.93} \mathrm{Y}_{0.07} \mathrm{O}_{3-\delta}$ by 4-probe dc measurements. J. Power Sources 142, 1-9 (2005).

43. Nomura, K. \& Kageyama, H. Transport properties of $\mathrm{Ba}\left(\mathrm{Zr}_{0.8} \mathrm{Y}_{0.2}\right) \mathrm{O}_{3-\delta}$ perovskite. Solid State Ion. 178, 661-665 (2007)

44. Traversa, E. Toward the miniaturization of solid oxide fuel cells. Interface 18 (3), 49-52 (2009). 
45. Yano, M., Tomita, A., Sano, M. \& Hibino, T. Recent advances in single-chamber solid oxide fuel cells: A review. Solid State Ion. 177, 3351-3359 (2007).

46. Ishihara, T., Shibayama, T., Honda, M., Nishiguchi, H. \& Takita, Y. Intermediate temperature solid oxide fuel cells using $\mathrm{LaGaO}_{3}$ electrolyte II. Improvement of oxide ion conductivity and power density by doping $\mathrm{Fe}$ for $\mathrm{Ga}$ site of $\mathrm{LaGaO}_{3}$. J. Electrochem. Soc. 147, 1332-1337 (2000).

47. Esposito, V. \& Traversa, E. Design of electroceramics for solid oxides fuel cell applications: Playing with ceria. J. Am. Ceram. Soc. 91, 1037-1051 (2008).

\section{Acknowledgements}

This work was partly supported by the Ministry of University and Research (MiUR) of Italy under the frame of the FISR project 'Polymer and Ceramic Electrolyte for Fuel Cells: System Validation and Development of New Materials', by the Ministry of Foreign Affairs (MAE) of Italy under the frame of the Italy-USA Joint Laboratory on 'Nanomaterials for Hydrogen and Sustainable Energy', and by the World Premier International Research

Center Initiative of MEXT, Japan. The authors would like to thank A. Chincarini for his helpful contribution for X-ray photoelectron spectroscopy analysis, and D. Marrè and E. Bellingeri for clarifying discussions and comments.

\section{Author contributions}

D.P. fabricated the films by PLD, carried out the XRD measurements, helped in the electrical measurements and wrote the manuscript together with E.F., who also carried out SEM observations, electrochemical measurements and analysed the data. A.D.E. and E.D.B. helped in the electrochemical measurements. A.T. and S.S. helped in thin-film fabrication and characterization. G.B. and S.L. supervised the work and revised the manuscript. E.T. was involved in study design, supervision of the work and manuscript revision.

\section{Additional information}

The authors declare no competing financial interests. Supplementary information accompanies this paper on www.nature.com/naturematerials. Reprints and permissions information is available online at http://npg.nature.com/reprintsandpermissions. Correspondence and requests for materials should be addressed to E.T. 